\title{
Co-administration of iloprost and eptifibatide in septic shock (CO-ILEPSS) - a randomised, controlled, double-blind investigator-initiated trial investigating safety and efficacy
}

Rasmus Ehrenfried Berthelsen ${ }^{1 *+}$ (D), Sisse Rye Ostrowski ${ }^{2,3+}$, Morten Heiberg Bestle ${ }^{1,3}$ and Per Ingemar Johansson ${ }^{4,5,6}$

\begin{abstract}
Background: Part of the pathophysiology in septic shock is a progressive activation of the endothelium and platelets leading to widespread microvascular injury with capillary leakage, microthrombi and consumption coagulopathy. Modulating the inflammatory response of endothelium and thrombocytes might attenuate this vicious cycle and improve outcome.
\end{abstract}

Method: The CO-ILEPSS trial was a randomised, placebo-controlled, double-blind, pilot trial. Patients admitted to the intensive care unit with septic shock were randomised and allocated in a 2:1 ratio to active treatment with dual therapy of iloprost $1 \mathrm{ng} / \mathrm{kg} / \mathrm{min}$ and eptifibatide $0.5 \mu \mathrm{g} / \mathrm{kg} / \mathrm{min}$ for $48 \mathrm{~h}$ or placebo. The primary outcomes were changes in biomarkers reflecting endothelial activation and disruption, platelet consumption and fibrinolysis. We compared groups with mixed models, post hoc Wilcoxon signed-rank test and Mann-Whitney $U$ test.

Results: We included 24 patients of which 18 (12 active, 6 placebo) completed the full 7-day trial period and were included in the per-protocol analyses of the primary outcomes. Direct comparison between groups showed no differences in the primary outcomes. Analyses of within-group delta values revealed that biomarkers of endothelial activation and disruption changed differently between groups with increasing levels of thrombomodulin $(p=0.03)$ and nucleosomes $(p=0.02)$ in the placebo group and decreasing levels of sE-Selectin $(p=0.007)$ and sVEGFR1 ( $p=0.005)$ in the active treatment group. Platelet count decreased the first $48 \mathrm{~h}$ in the placebo group $(p=0.049)$ and increased from baseline to day 7 in the active treatment group $(p=0.023)$. Levels of fibrin monomers declined in the active treatment group within the first $48 \mathrm{~h}(p=0.048)$ and onwards $(p=0.03)$. Furthermore, there was a significant reduction in SOFA score from $48 \mathrm{~h}(p=0.024)$ and onwards in the active treatment group.

Intention-to-treat analyses of all included patients showed no differences in serious adverse events including bleeding, use of blood products or mortality.

Conclusion: Our results could indicate benefit from the experimental treatment with reduced endothelial injury, reduced platelet consumption and ensuing reduction in fibrinolytic biomarkers along with improved SOFA score. The results of the CO-ILEPSS trial are exploratory and hypothesis generating and warrant further investigation in a large-scale trial.

(Continued on next page)

\footnotetext{
* Correspondence: rab@regionsjaelland.dk

${ }^{\dagger}$ Rasmus Ehrenfried Berthelsen and Sisse Rye Ostrowski contributed equally

to this work

${ }^{1}$ Department of Anesthesiology and Intensive Care, Zealand University

Hospital, Roskilde, University of Copenhagen, Copenhagen, Denmark

Full list of author information is available at the end of the article
}

(c) The Author(s). 2019 Open Access This article is distributed under the terms of the Creative Commons Attribution 4.0 International License (http://creativecommons.org/licenses/by/4.0/), which permits unrestricted use, distribution, and reproduction in any medium, provided you give appropriate credit to the original author(s) and the source, provide a link to the Creative Commons license, and indicate if changes were made. The Creative Commons Public Domain Dedication waiver (http://creativecommons.org/publicdomain/zero/1.0/) applies to the data made available in this article, unless otherwise stated. 


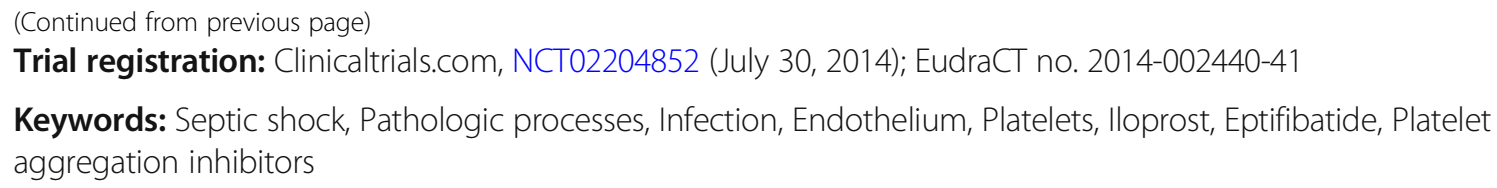

\section{Introduction}

Septic shock is a leading cause of death in the intensive care unit (ICU) with mortality rates above $40 \%[1,2]$. Treatment strategies consist of early recognition and diagnosis to facilitate timely initiation of antibiotic therapy and supportive care [3]. A series of pathogenic events are responsible for the transition from sepsis to septic shock. The initial reaction to infection is a neurohumoral, generalised pro- and anti-inflammatory response $[4,5]$ resulting in mobilisation and/or "spill over" of plasma substances and excessive cellular, coagulation and endothelial activation. The proinflammatory response induces widespread endothelial and microvascular injury resulting in disseminated intravascular coagulation with microvascular thrombosis, consumptive thrombocytopenia, coagulopathy, bleeding and a loss of endothelial integrity ultimately leading to capillary leakage, tissue oedema, tissue ischaemia and shock [5-7]. In the later stages of sepsis, immunodeficiency is a critical component of the pathology that causes multiple organ failure and death [8].

There are three major pathogenic pathways associated with the coagulopathy in sepsis: (1) tissue factor-mediated thrombin generation, (2) dysfunctional anticoagulant pathways and (3) blocked fibrinolysis [9]. Treatment strategies aimed at reducing coagulation activation with antithrombin [10], tissue factor pathway inhibitor [11] and activated Protein $C[12,13]$ have all failed to show improved survival in large clinical trials refuting this as a pathophysiological explanation.

The platelets and endothelium are interdependent in the vicious cycle of endothelial damage, microcirculatory failure, consumptive thrombocytopenia, coagulopathy, bleeding, immunodeficiency, tissue ischaemia, shock, organ failure and death, in patients with severe sepsis/septic shock. Selective targeting of either platelets or the endothelium may be sufficient to prevent the progressively more activated and damaged endothelium and activation of the platelets [14].

Prostacyclin is an endogenously produced molecule with anti-platelet, vasodilatory and cytoprotective properties released from the healthy endothelium as part of the natural anticoagulation system [15]. Intravenous prostacyclin in doses of $0.5-2.0 \mathrm{ng} / \mathrm{kg} / \mathrm{min}$ has been reported to be successful at achieving endothelial modulating/preserving effects in patients with traumatic brain injury, without significant haemodynamic or platelet aggregation complications [16, 17].
Eptifibatide is a platelet glycoprotein (GP) IIb/IIIa receptor inhibitor that prohibits clot development in a predictable and easy controllable way. Inhibition of the GPIIb/IIIa receptor does not alter the paracrine function of platelets, which is considered a crucial part of maintaining vascular integrity and preventing haemorrhage in conditions with inflammation $[18,19]$. Animal studies have reported that treatment with GPIIb/IIIa inhibitor protects against endothelial dysfunction in experimental endotoxemia [20, 21]. Furthermore, casuistic findings have shown that GPIIb/IIIa inhibition leads to clinically relevant thrombolysis in patients with mechanical prosthetic mitral valve thrombosis $[22,23]$.

The objective of the CO-ILEPSS trial was to investigate safety and efficacy of a combined infusion of lowdose prostacyclin (iloprost) and GPIIb/IIIa inhibitor (eptifibatide) for $48 \mathrm{~h}$ in patients with septic shock. We hypothesised that this dual treatment with iloprost and eptifibatide would deactivate the endothelium and restore vascular integrity, reduce formation of microvascular thrombosis and dissolve existing thrombi in the microcirculation and maintain platelet counts leading to improved platelet-mediated immune function and reduced risk of bleeding. Compared to the standard treatment (placebo), this was expected to translate into reduced organ failure and improved outcome in patients with septic shock.

\section{Methods \\ Design}

The CO-ILEPSS trial was an investigator-initiated singlecentre randomised, placebo-controlled, double-blind phase 2a trial in patients with septic shock.

The trial was conducted from October 2014 to May 2016, and there were no significant changes to the trial protocol during the course of the trial. The trial is reported in accordance with the CONSORT statement [24], and a populated CONSORT checklist is available in Additional file 1. The trial was approved by the regional ethics committee, and all patients and/or their next of kin gave informed consent to participate. The full trial protocol is available in Additional file 2.

\section{Participants}

Patients were allocated in a 2:1 ratio with 15 intentionto-treat (ITT) patients allocated to active treatment and 9 ITT patients allocated to control treatment (placebo). Patients who dropped out or were withdrawn from the 
trial prior to day 7 were replaced to ensure adequate data points, and 12 active treatment and 6 placebo patients, respectively, were treated as per protocol (PP). To replace patients, who were withdrawn, unblinded trial personal added envelopes containing the same allocation as the ones who dropped out and we recruited and rerandomised new patients.

We screened patients admitted to the intensive care unit (ICU) at Nordsjaellands Hospital (NOH) during the inclusion period. Patients were screened within $24 \mathrm{~h}$ of admission according to the following inclusion criteria:

- Adult intensive care patients (age $\geq 18$ years)

- Sepsis, defined as suspected or confirmed site of infection or positive blood culture and $\geq 2$ of 4 systemic inflammatory response syndrome (SIRS) criteria fulfilled within the last $24 \mathrm{~h}$ :

a. Temperature $\leq 36{ }^{\circ} \mathrm{C}$ or $\geq 38^{\circ} \mathrm{C}$

b. Heart rate $\geq 90$ beats per minute

c. Mechanical ventilation for acute respiratory process or respiratory rate $\geq 20$ breaths per minute or $\mathrm{PaCO} 2<4.2 \mathrm{kPa}$

d. $\quad W B C \geq 12,000 / \mathrm{mm} ; \mathrm{OR} \leq 4000 / \mathrm{mm}$ OR $>10 \%$ bands

- Septic shock within the last $24 \mathrm{~h}$, defined as:

a. Hypotension (MAP $<70 \mathrm{mmHg}$, lactate $4 \mathrm{mmol} /$ l) despite ongoing resuscitation with fluids (crystalloids, colloids, blood products) within the last $24 \mathrm{~h}$ or

b. $\geq 30 \mathrm{ml} / \mathrm{kg}$ ideal body weight (IBW) fluid (crystalloids, colloids, blood products) given in the last $24 \mathrm{~h}$ and

c. Need for vasopressor/inotropic agents (noradrenaline, adrenaline or dopamine) within the last $24 \mathrm{~h}$

- Can be randomised into trial and dosed $<24 \mathrm{~h}$ after septic shock diagnosis (the time point for the septic shock diagnosis corresponds to the time point where the vasopressor/inotropic therapy is initiated) and

- Consent is obtainable

A full description of the inclusion and exclusion criteria is provided in Additional file 3.

\section{Randomisation}

The random allocation sequence was computer generated, and allocation pages were packed in sealed opaque envelopes. The envelopes were prepared by the principal investigator (SRO) at Rigshospitalet (RH) and delivered at the trial site $\mathrm{NOH}$. At $\mathrm{NOH}$, the envelopes were stored in a locked office at the post-anaesthesia care unit (PACU) located in a separate building from the ICU. The local investigators (REB and MHB) did not have access to this office. When a patient fulfilled inclusion criteria and consent had been obtained, randomisation was done by placing a phone call from the ICU to a nurse at the PACU. The nurse then opened the next envelope in line and prepared the trial drug or placebo according to the instructions. Syringes containing trial drug or placebo drug were then delivered to the investigator (REB) at the ICU where trial treatment was initiated.

\section{Intervention}

Patients allocated to the active treatment arm received dual infusions of prostacyclin (iloprost) $1.0 \mathrm{ng} / \mathrm{kg} / \mathrm{min}$ and GPIIb/IIIa inhibitor (eptifibatide) $0.5 \mu \mathrm{g} / \mathrm{kg} / \mathrm{min}$ for $48 \mathrm{~h}$. Iloprost and eptifibatide were both diluted in saline to a concentration with which the targeted treatment was achieved with an infusion of $4 \mathrm{ml} / \mathrm{h}$. Treatment in the placebo group consisted of dual infusions of normal saline $4 \mathrm{ml} / \mathrm{h}$ for $48 \mathrm{~h}$. The infusions of both active and placebo treatment were given either in two separate legs of a central venous catheter or in two separate peripheral venous catheters.

\section{Outcomes}

The primary outcome of the CO-ILEPSS trial was divided in three different measures. These were:

1) Change in biomarkers indicative of endothelial activation and damage (sE-selectin, syndecan-1, soluble thrombomodulin (sTM), sVE-cadherin, nucleosomes, vascular endothelial growth factor (VEGF) and soluble vascular endothelial growth factor receptor 1 (sVEGFR1)) from baseline to $48 \mathrm{~h}$ post-randomisation

2) Change in platelet count from baseline to $48 \mathrm{~h}$ post-randomisation

3) Change in D-dimer and fibrin split products indicative of fibrinolysis (fibrin monomer complex, fibrin degradation products, D-dimer) from baseline to $48 \mathrm{~h}$ post-randomisation.

The reason for having three primary sub-endpoints was that they reflect different effects of active treatment on the vascular system that we wished to evaluate, i.e. endothelial activation, platelet consumption and fibrinolysis.

Secondary outcomes included severe bleeding (intracranial or clinical bleeding with the use of 3 RBC units or more/24 h); use of blood products in the ICU post-randomisation; mortality at days 7, 30 and 90; changes in Sequential Organ Failure Assessment (SOFA) score from baseline; and days of vasopressor, mechanical ventilation and renal replacement therapy (RRT) post-randomisation. 


\section{Sample size/power calculation}

The sample size for the CO-ILEPSS trial was not based upon a power calculation because there were no available data on the specific active dual drug therapy vs placebo in patients with septic shock.

However, in a previous study of safety and efficacy of prostacyclin vs placebo in patients undergoing Whipple surgery, post-operative levels of sVE-cadherin increased $1978 \pm 461 \mathrm{pg} / \mathrm{ml}$ in the placebo group [25]. Based on this, we would be able to detect a difference of $33 \%$ in sVE-cadherin increase between groups with 12 patients in the active treatment group vs 6 patients in the placebo group, with a power of 0.8 and an alfa of 0.05 .

\section{Blinding}

The CO-ILEPSS trial was a double-blind, placebo-controlled trial, and all participants, next of kin, caregivers, investigators and sponsors were blinded for the trial allocation.

Both trial medications were colourless when diluted in saline, and it was impossible to distinguish the syringes with trial medicine from those with saline. Since the number of patients in the different groups was unequal, it was not possible to maintain blinding during the statistical analyses, but these were conducted according to the statistical analysis plan generated as part of the trial protocol.

\section{Biomarker analyses}

Blood samples for the analyses of biomarkers were drawn pre-study drug/placebo administration, post $6 \mathrm{~h}, 24 \mathrm{~h}, 48$ $\mathrm{h}, 72 \mathrm{~h}$ and $120 \mathrm{~h}$. All samples were transferred to the local Blood Bank at the trial site for further processing (centrifugation, plasma aliquoting and freezing) and stored at $80^{\circ} \mathrm{C}$ for later analyses at the Dept. of Clinical Immunology, Blood Bank, Copenhagen University Hospital, Rigshospitalet. In brief, biomarkers were analysed by commercially available enzyme-linked immunosorbent assay (ELISA) according to the manufacturer's recommendations: Syndecan-1 (Diaclone SAS, Besancon, France; lower limit of detection (LLD) $4.94 \mathrm{ng} / \mathrm{ml}$ ), soluble thrombomodulin (sTM, Diaclone SAS, Besancon, France; LLD 0.31 $\mathrm{ng} / \mathrm{ml}$ ), soluble E-selectin (sE-selectin, sCD62E, Diaclone SAS, Besancon, France; LLD $<0.5 \mathrm{ng} / \mathrm{ml}$ ), vascular endothelial (VE) cadherin (VE-cadherin, R\&D Systems, Europe, Ltd., Abingdon, UK; LLD $0.113 \mathrm{ng} / \mathrm{ml}$ ), vascular endothelial growth factor (VEGF, R\&D Systems, Europe, Ltd., Abingdon, UK; LLD $9 \mathrm{pg} / \mathrm{ml}$ ), histone-associated DNA fragments (nucleosomes, Cell Death Detection ELISA ${ }^{\text {PLUS }}$, Roche Diagnostics GmbH, Mannheim, Germany; LLD relative percentage with a maximal standard included), fibrin monomer complex (Biocompare, LLD $1.56 \mu \mathrm{g} / \mathrm{ml}$ ); fibrin degradation product (Cloud-Clone Corp., Houston, USA; LLD $0.69 \mathrm{ng} / \mathrm{ml}$ ), and D-dimer (Sekisui Diagnostics, LLC, Stanford, CT, USA; LLD 2-4 ng/ml).

\section{Statistical analysis}

Summary statistics of continuous variables are presented as median with interquartile range (IQR). Summary statistics of frequency tables are presented as $n(\%)$. $p$ values < 0.05 are considered significant.

The primary outcomes were analysed for efficacy in PP analyses. The difference between treatment groups for continuous data was evaluated with the analysis of variance (mixed model) and post hoc pairwise comparisons of means. Furthermore, delta values (numerical change in variables between time points) within and between groups were compared by paired (Wilcoxon signed-rank test) and non-paired (Mann-Whitney $U$ test) non-parametric tests.

Biomarker measurements are presented as absolute values in Figs. 2 and 3 and as relative changes in percentage from baseline in Additional file 4.

Secondary outcomes were analysed on an ITT basis. The differences between treatment groups for categorical data were evaluated with McNemar's test (change over time), frequency tables and chi-square statistics. The difference between treatment groups for continuous data was evaluated using the analysis of variance (mixed model) followed by post hoc pairwise comparisons of means. If the assumption of normality was not fulfilled, non-parametric test and Wilcoxon rank-sum test were used. Statistical analysis was performed using SAS 9.1.3 SP4 (SAS Institute Inc., Cary, NC, US).

\section{Results}

During the study period, we screened 509 patients and included 24. Most patients were excluded due to the absence of septic shock or completed/scheduled surgery within $\pm 48 \mathrm{~h}$. Of the included patients, two patients were withdrawn prior to initiation of trial treatment, and four patients were withdrawn prior to day 7 (Fig. 1). These six patients were replaced in the trial. Reasons for withdrawal were incorrect inclusion (1), emergency surgery (1), transfer to another ICU (1), therapeutic anticoagulation therapy (2) and treatment with inhaled prostacyclin (1).

Table 1 shows baseline characteristics, use of organ supportive therapy and outcomes of patients included in the PP analyses. Only alkaline phosphatase was significantly different between groups at baseline, and it is worth noting that the disease severity was considerable with SOFA scores of 8-10, SAPS II scores of 46-48 and an observed 90 -day mortality of $25-50 \%$.

\section{Primary outcomes}

The PP primary analysis included data from the 18 patients (12 active and 6 placebo) who completed the full 7 days of the trial. 


\section{CONSORT \\ TRANSPARENT REPORTING Of TRIALS \\ CONSORT 2010 Flow Diagram}

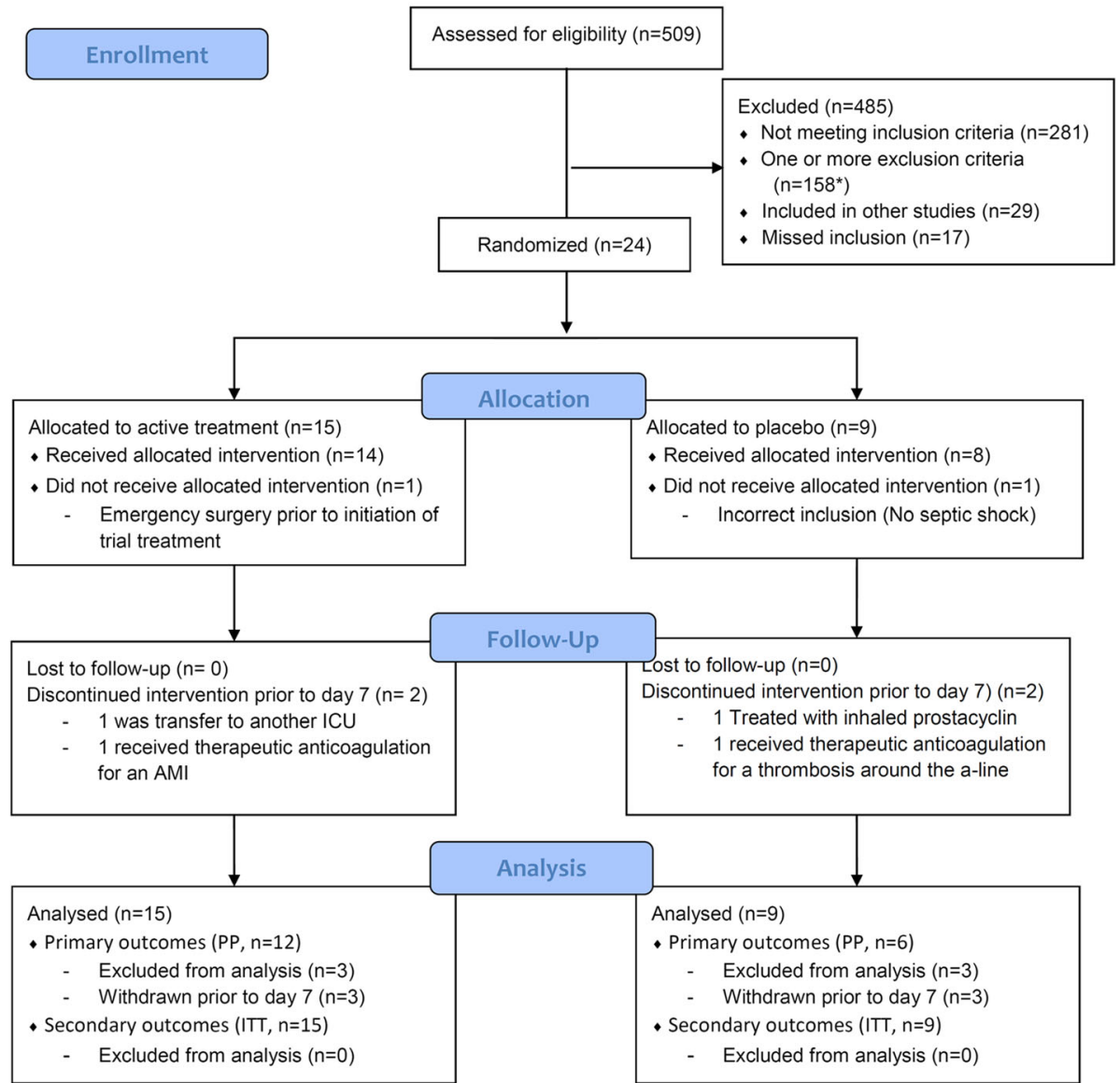

* Surgery within $48 \mathrm{~h}(n=76)$, Do not resuscitate order $(n=24)$, Chronic Dialysis $(n=20)$, Therapeutic antithrombotic treatment $(n=27)$, Traumatic Brain Injury $(n=2)$, Bleeding with need for blood products within $24 \mathrm{~h}(n=3), B W>125 \mathrm{~kg}(n=2)$, pulmonary hypertension ( $N=2)$, Contraindication for antithrombotic therapy ( $n=1)$, Platelet count $<10$ ( $n=1)$

Fig. 1 Consort flow diagram. ICU intensive care unit, AMI acute myocardial infarction, A-line arterial line, PP per protocol, ITT intention to treat

\section{Endothelial disruption biomarkers}

At baseline, sVE-cadherin was significantly higher in the placebo group $(p=0.047)$ (Fig. 2a). Apart from this, there were no differences in the measured biomarkers between groups at baseline or at any time point during the 5-day follow-up (Fig. 2a-f). There were, however, differences in the within-group changes over time: At 6 $\mathrm{h}$, there was a significant increase in both $\operatorname{sTM}(p=$ 0.03 ) and nucleosomes $(p=0.02)$ (Fig. $2 \mathrm{~b}, \mathrm{c})$ only in the placebo group. Furthermore, in the placebo group, there was a tendency towards increasing levels of nucleosomes for up to $72 \mathrm{~h}(p=0.06)$ (Fig. 2c).
At $48 \mathrm{~h}$ and throughout day 5, there was a significant decrease in sE-Selectin $(p=0.007)$ and sVEGFR1 $(p=0.005)$ only in the active treatment group (Fig. $2 \mathrm{~d}, \mathrm{e}$ ).

\section{Platelet count}

The platelet count did not differ significantly between groups at any time point during the trial. Similarly to the endothelial disruption biomarkers, there were differences in the within-group changes over time with a decline from baseline to $48 \mathrm{~h}$ only in the placebo group $(p=0.049)$ and an increase from baseline to day 7 only in the active treatment group $(p=0.023)$ (Fig. 3a). 
Table 1 Baseline characteristics, use of organ support and outcome of patients included in the per-protocol analyses

\begin{tabular}{|c|c|c|}
\hline \multirow[t]{2}{*}{ Variable } & $\begin{array}{l}\text { Active } \\
\text { treatment }\end{array}$ & $\begin{array}{l}\text { Placebo } \\
\text { treatment }\end{array}$ \\
\hline & Median (IQR) & Median (IQR) \\
\hline$n$ & 12 & 6 \\
\hline Age, years & $61.5(54.2-68.6)$ & $71.3(64.2-75)$ \\
\hline Male gender, $n(\%)$ & $8(66.7 \%)$ & $6(100 \%)$ \\
\hline $\mathrm{BMl}, \mathrm{kg} / \mathrm{m}^{2}$ & $24.5(22.1-28.7)$ & $27.8(22.8-30.4)$ \\
\hline \multicolumn{3}{|l|}{ Infectious focus } \\
\hline CNS, $n(\%)$ & $0(0 \%)$ & $0(0 \%)$ \\
\hline Lungs, $n(\%)$ & $10(83.3 \%)$ & $4(66.7 \%)$ \\
\hline Gastrointestinal, $n(\%)$ & $0(0 \%)$ & $2(33.3 \%)$ \\
\hline Urogenital, $n(\%)$ & $0(0 \%)$ & $0(0 \%)$ \\
\hline Skins and soft tissue, $n(\%)$ & $2(16.7 \%)$ & $1(16.7 \%)$ \\
\hline Blood, $n(\%)$ & $0(0 \%)$ & $1(16.7 \%)$ \\
\hline Unknown, $n(\%)$ & $1(8.3 \%)$ & $0(0 \%)$ \\
\hline $\begin{array}{l}\text { Identified pathogen } \\
\text { (microbiology), } n(\%)\end{array}$ & $9(75 \%)$ & $5(83.3 \%)$ \\
\hline \multicolumn{3}{|l|}{ Comorbidity } \\
\hline Chronic diagnosis, $n$ (\%) & $9(75 \%)$ & $6(100 \%)$ \\
\hline \multicolumn{3}{|l|}{ Admitted from } \\
\hline Acute medical care unit, $n(\%)$ & $6(50 \%)$ & $3(50 \%)$ \\
\hline Medical department, $n(\%)$ & $3(25 \%)$ & $1(16.7 \%)$ \\
\hline \multicolumn{3}{|c|}{ Disease severity, physiology and biochemistry } \\
\hline SOFA (first) & $9.0(7.8-12.0)$ & $9.5(7.3-13.3)$ \\
\hline SAPS ॥ & $46(42-57)$ & $48(39-55)$ \\
\hline Systolic blood pressure, $\mathrm{mmHg}$ & $113(100-120)$ & $123(104-145)$ \\
\hline Lactate, mmol/l & $1.4(1.3-3.1)$ & $3.5(1.9-3.9)$ \\
\hline $\mathrm{pH}$ & $7.35(7.3-7.41)$ & $7.41(7.39-7.41)$ \\
\hline Haemoglobin, mmol/l & $6.9(6.4-7.7)$ & $6.5(5.8-7)$ \\
\hline White blood cell count, $\times 10^{9} / \mathrm{I}$ & $15(9.8-19.1)$ & $17.2(8.8-23.1)$ \\
\hline Platelet count, $\times 10^{9} / 1$ & $188(130-255)$ & $212(149-295)$ \\
\hline INR & $1.2(1.1-1.2)$ & $1.3(1.1-2)$ \\
\hline Antithrombin & $0.64(0.52-0.76)$ & $0.58(0.37-0.82)$ \\
\hline D-dimer & $4.6(2.4-19.9)$ & $14.4(3.4-15.3)$ \\
\hline Creatinine, $\mu \mathrm{mol} / /$ & $97(67-242)$ & $126(114-189)$ \\
\hline Blood urea nitrogen (BUN), $\mathrm{mmol} / \mathrm{l}$ & $7.9(5.3-13.2)$ & $13.5(8.6-17.9)$ \\
\hline ALAT, U/I & $35(26-62)$ & $35(23-169)$ \\
\hline Bilirubin, $\mu \mathrm{mol} / \mathrm{l}$ & $11(5-24)$ & $16(8-29)$ \\
\hline Basic phosphatase, U/l & $64(57-84)$ & $149(76-242)$ \\
\hline C-reactive protein, mg/l & $172(129-244)$ & 138 (89-169) \\
\hline \multicolumn{3}{|l|}{ Therapy and outcome } \\
\hline Ventilator days, $n(\%)$ & $5(3-6)$ & $6(4-6)$ \\
\hline Ventilator-free days, $n$ (\%) & $25(24-27)$ & $24(6-24)$ \\
\hline RRT days, n (\%) & $0(0-0)$ & $0(0-0)$ \\
\hline RRT-free days, $n$ (\%) & $30(29-30)$ & $30(8-30)$ \\
\hline
\end{tabular}

Table 1 Baseline characteristics, use of organ support and outcome of patients included in the per-protocol analyses (Continued)

\begin{tabular}{lll}
\hline Variable & $\begin{array}{l}\text { Active } \\
\text { treatment } \\
\text { Median (IQR) }\end{array}$ & $\begin{array}{l}\text { Placebo } \\
\text { treatment } \\
\text { Median (IQR) }\end{array}$ \\
\hline Vasopressor days, $n$ (\%) & $5(5-5)$ & $5(4-5)$ \\
Vasopressor-free days, $n$ (\%) & $25(24-25)$ & $25(6-25)$ \\
Discharged to admitting dept., $n$ (\%) & $9(75 \%)$ & $3(50 \%)$ \\
Discharged to other hosp., $n(\%)$ & $1(8.3 \%)$ & $1(16.7 \%)$ \\
Discharged to other ICU, $n(\%)$ & $1(8.3 \%)$ & $1(16.7 \%)$ \\
Active stop therapy, $n(\%)$ & $2(16.7 \%)$ & $1(16.7 \%)$ \\
7-day mortality, $n(\%)$ & $0(0 \%)$ & $1(16.7 \%)$ \\
30-day mortality, $n(\%)$ & $1(8.3 \%)$ & $2(33.3 \%)$ \\
90-day mortality, $n(\%)$ & $3(25 \%)$ & $3(50 \%)$ \\
\hline
\end{tabular}

$I Q R$ interquartile range, $B M I$ body mass index, CNS central nervous system, SOFA Sequential Organ Failure Assessment, SAPS // Simplified Acute Physiology Score II, INR international normalised ratio, ALAT alanine aminotransferase, RRT renal replacement therapy, ICU intensive care unit, NS non-significant

\section{Fibrinolytic biomarkers}

D-dimer and fibrinogen degradation products were similar in both groups (Fig. 3b, c). Levels of fibrin monomers were higher in the active treatment group than in the placebo group at baseline. Comparison of within-group delta values showed a significant decline within the first $48 \mathrm{~h}(p=0.048)$ and onwards only in the active treatment group (time effect $p=0.04$ ) (Fig. $3 \mathrm{~d}$ ).

\section{Secondary outcomes}

In the ITT secondary analyses, a total of 24 patients (15 active and 9 placebo) were included.

All secondary endpoints including safety measures of bleeding, use of blood products in the ICU and mortality were comparable between groups (Additional file 5). Additionally, there was no difference in the occurrences of serious adverse events (SAEs) between groups and there were no suspected unexpected serious adverse reactions (SUSARs) in either group. Occurrences of SAEs and reasons for withdrawal and exclusions are summarised in Table 2.

Comparison of within-group changes over time revealed a significant reduction in SOFA score at $48 \mathrm{~h}$ $(p=0.024)$ and onwards in the active treatment group, but not in the placebo group.

\section{Discussion}

\section{Main findings}

Dual therapy with iloprost and eptifibatide for $48 \mathrm{~h}$ in patients with septic shock had no significant effect on absolute values of biomarker levels compared to placebo treatment.

Analysis of within-group changes over time revealed biomarker changes indicative of reduced endothelial 

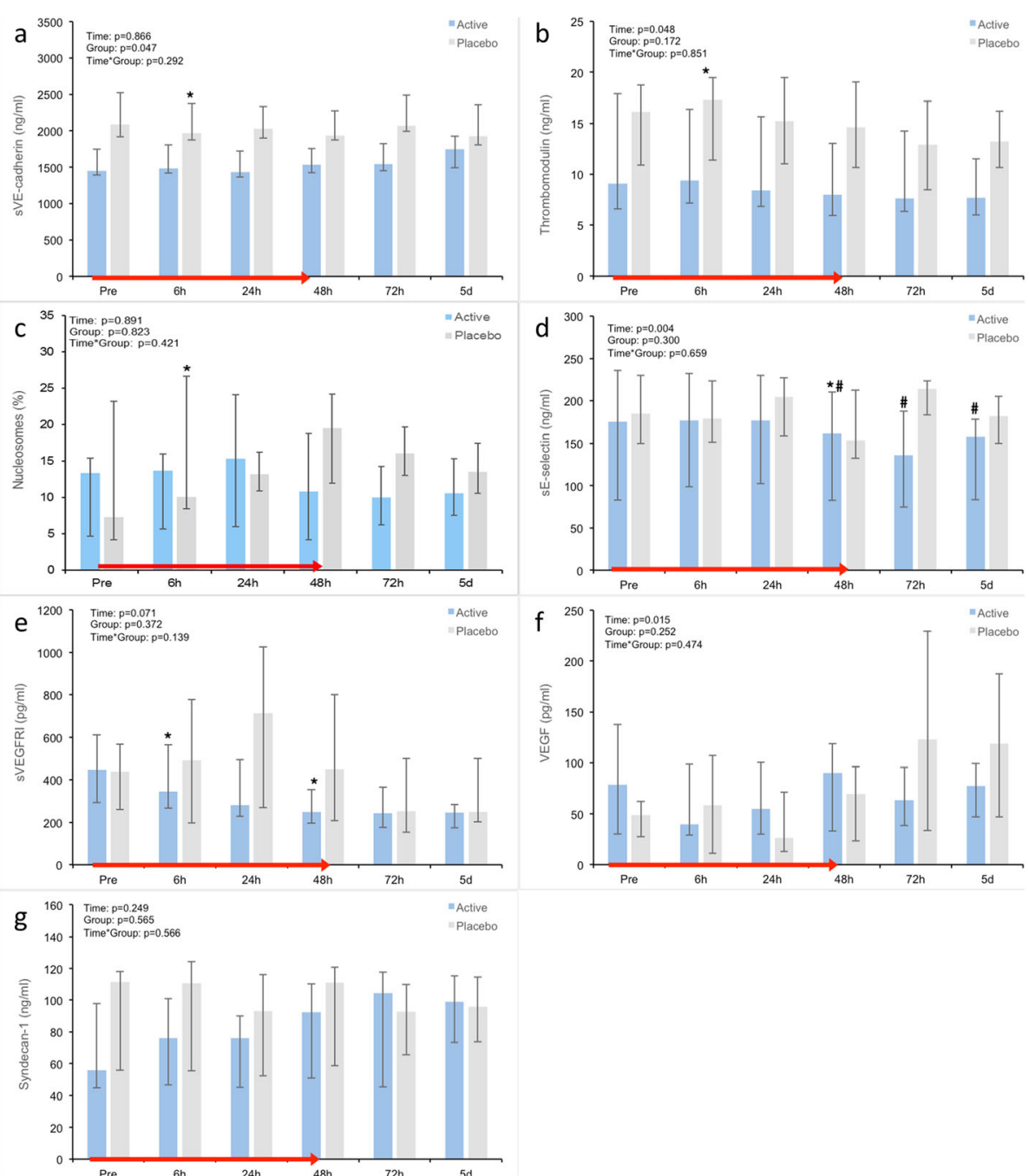

Fig. 2 a-g Comparison of time-dependent changes in absolute biomarker values. Data shown as median (IQR). *Within-group change from baseline, $p<0.05$; " within-group change from non-baseline, $p<0.05$. SVEGFRI soluble vascular endothelial growth factor receptor 1

injury, reduced fibrinolysis and reduced platelet consumption in the active treatment group. This was accompanied by an overall decline in SOFA score from 48 $h$ and onwards that was not observed in the placebotreated patients. Additionally, the trial treatment was not associated with increased bleeding or the use of blood products as compared to placebo.

\section{Endothelial injury}

Taken together, the observed changes in STM, nucleosomes, sE-selectin, VEGF and sVEGFR1 could reflect reduced levels of endothelial activation, disruption and cell damage.

Prostacyclin doses, corresponding to the low dose chosen for this trial $(1.0 \mathrm{ng} / \mathrm{kg} / \mathrm{min})$, have previously been demonstrated not to increase bleeding risk or haematoma size in patients with traumatic brain injury [17] and to reduce the need for blood transfusion in patients undergoing Whipple surgery due to pancreatic cancer [25]. Our results are in alignment with these former trials which also demonstrated beneficial effects of iloprost on vascular integrity in critically ill patients reflected by similar changes in SE-selectin, sTM and nucleosomes [17, 25, 26].

Endothelial protection could be ascribed to the cytoprotective effects of prostacyclin, which in its endogenous form induces a reduction in inflammation and stabilisation of lysosomal and cell membranes [27]. The dose of $1.0 \mathrm{ng} / \mathrm{kg} / \mathrm{min}$ is approximately five- to tenfold higher than the normal endogenous production of prostacyclin from the healthy endothelium [28], and we expected this to restore vascular integrity in septic patients with endothelial injury and dysfunction. 


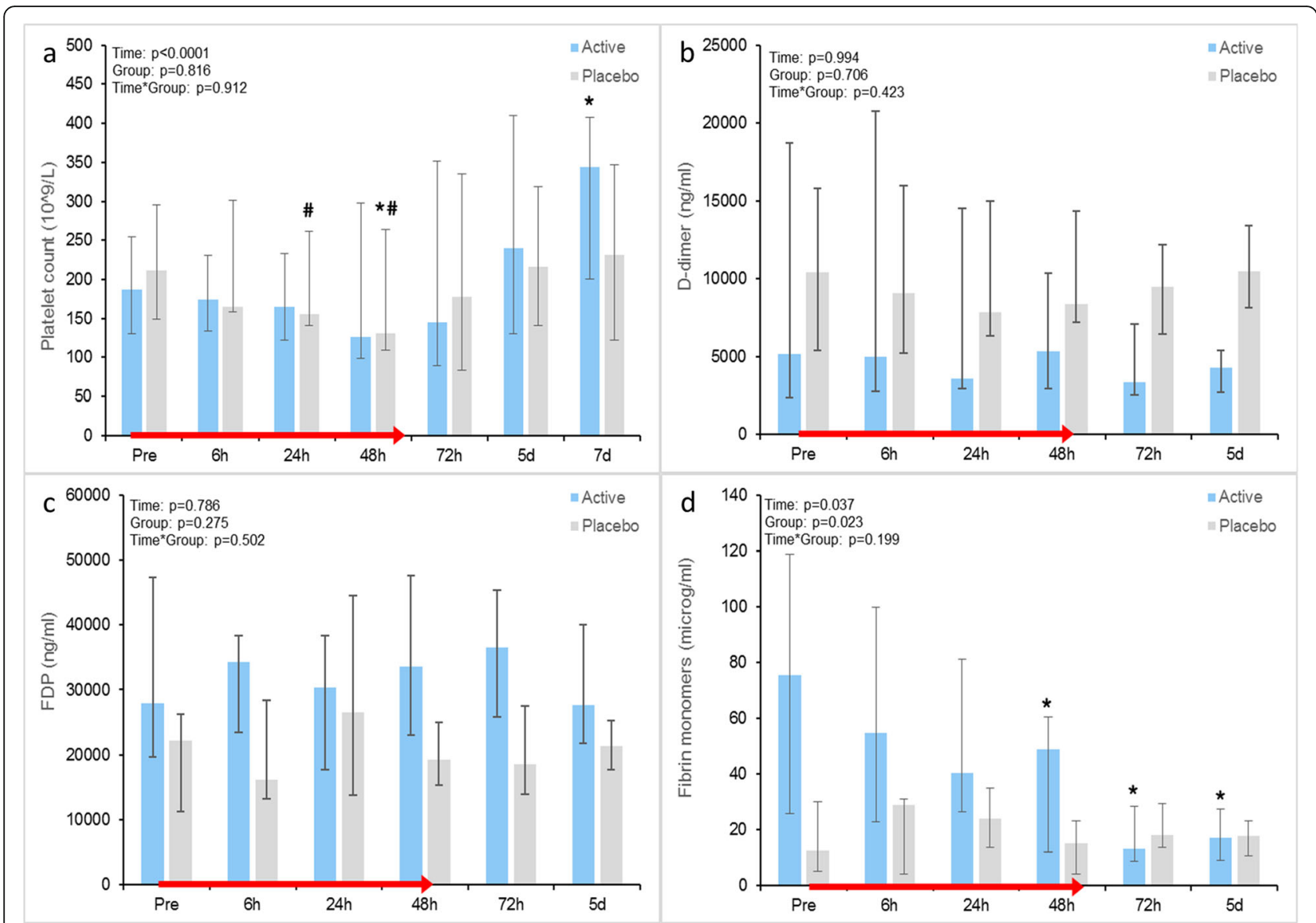

Fig. 3 a-d Comparison of time-dependent changes in platelet count, D-dimer, Fibrin monomers and SOFA score. Data shown as median (IQR). *Within-group change from baseline, $p<0.05$; "within-group change from non-baseline, $p<0.05$. SOFA Sequential Organ Failure Assessment

Table 2 SAE/withdrawal/exclusion

\begin{tabular}{|c|c|c|c|}
\hline ITT/PP & $\begin{array}{l}\text { Allocation } \\
\text { [duration] }\end{array}$ & SAE/withdrawal/exclusion & Outcome \\
\hline ITT & Placebo $[0 \mathrm{~h}]$ & $\begin{array}{l}\text { Recovery with extubation before trial drug administration (incl. criteria not met). } \\
\text { Excluded }\end{array}$ & Death on day 13 (resp. failure) \\
\hline ITT & Placebo $[14 \mathrm{~h}]$ & $\begin{array}{l}\text { Thrombosis in the arterial cannula (difficult insertion in Aa. radialis, instead US-guided } \\
\text { insertion in a. brachialis). SAE/withdrawal }\end{array}$ & $\begin{array}{l}\text { LMWH treatment ( } 10.000 \mathrm{IE}) \\
\text { with good effect. Survivor }\end{array}$ \\
\hline ITT & Placebo $[14 \mathrm{~h}]$ & Indication for Flolan inhalation (severe respiratory failure). SAE/withdrawal & $\begin{array}{l}\text { Death on day } 2 \\
\text { (respiratory failure) }\end{array}$ \\
\hline PP & Placebo [completed] & $\begin{array}{l}\text { latrogenic pneumothorax and intraabdominal bleeding after liver abscess drainage, } 28 \mathrm{~h} \\
\text { after ceasing trial drug. SAE }\end{array}$ & Death on day 2 (pneumonia) \\
\hline ITT & Active $[0 \mathrm{~h}]$ & Indication for acute abdominal surgery before trial drug administration. Excluded & Survivor \\
\hline ITT & Active $[21 \mathrm{~h}]$ & $\begin{array}{l}\text { Indication for therapeutic LMWH (suspicion of type II MI). Cardiac enzymes elevated before } \\
\text { inclusion, but increasing. SAE/withdrawal }\end{array}$ & $\begin{array}{l}\text { Death on day } 10 \\
\text { (cardiac failure) }\end{array}$ \\
\hline ITT & Active $[28 \mathrm{~h}]$ & Transferred to other ICU due to overcrowding. Withdrawal & Survivor \\
\hline PP & Active [complete] & $\begin{array}{l}\text { Severe septic shock (Capnocytophagus carnimorsus) with bradycardia treated with } 1 \mathrm{mg} \\
\text { adrenalin IV and } 2 \text { min CPR. Circulatory stabilised after } 2 \text { min. Transferred to tertiary ICU } \\
\text { day } 4 \text {. Necrotomy of the face and leg day 27. Finger amputation (1., 2., 4., 5. finger on } \\
\text { the left hand) day 35. Femur amputation day 50. SAE }\end{array}$ & Survivor \\
\hline
\end{tabular}


The clinical impact of within-group changes in these biomarkers remains to be seen, but observational data have linked increased levels of sTM to reduced survival in patients with septic shock [29].

\section{Platelet protection and thrombotic activity}

The increasing platelet count in the active treatment group could indicate protective effect against platelet consumption. In a previous study by Link et al., it was demonstrated that administration of a platelet GPIIb/IIIa receptor inhibitor in combination with unfractionated heparin for $96 \mathrm{~h}$ was tolerated in patients with cardiogenic shock and need for dialysis. Importantly, in this study, treatment with the GPIIb/IIIa receptor inhibitor was not associated with increased bleeding but was rather associated with a significantly lower number of platelet transfusions and a higher, maintained platelet count, as compared to controls anticoagulated with heparin alone [30]. This finding of preserved platelet count is in accordance with the finding in our study.

In addition to preserved platelet counts, the inhibition of platelet-monocyte aggregation might have an anti-inflammatory effect, and thus serve to protect the endothelium and microvasculature [31]. A reduced thrombotic activity was reflected in declining levels of fibrin monomers in the active treatment group.

\section{Safety}

The individual doses of iloprost and eptifibatide selected for this trial are lower than the recommended doses for their respective approved indications. The dosages chosen for the current trial are in alignment with doses that have been reported to result in the desired effect for each agent without causing significant adverse side effects $[17,25,30]$.

The safety of the co-administration of eptifibatide and iloprost in a dose comparable to the dose applied in the present study (eptifibatide $0.5 \mu \mathrm{g} / \mathrm{kg} / \mathrm{min}+$ iloprost 1.0 $\mathrm{ng} / \mathrm{kg} / \mathrm{min}$ infused for $24 \mathrm{~h}$ ) is supported by a completed phase I/II trial in patients undergoing primary PCI due to ST-elevated myocardial infarct [26]. This trial found no bleeding-related adverse events, and no treatment-related adverse events occurred.

\section{Limitations}

Our main inclusion criterion was septic shock defined as the use of norepinephrine in patients with sepsis. This ensured that the screening and inclusion process was rather pragmatic and easy to perform, but we might have limited the potential effect of our intervention, since it is not given that all patients with septic shock have equal degrees of endothelial dysfunction and/or consumption coagulopathy. If we had used one or more specific markers in our screening, we might have been able to show higher efficacy of our trial treatment.

The CO-ILEPSS pilot trial is exploratory and hypothesis generating. Our small sample size and the single-centre design limit both external validity and the ability to draw any definitive conclusions from our results. The lack of a power calculation might have limited our ability to detect a difference in our primary outcome. Furthermore, our primary outcome is a composite of three categories of biomarkers with a total of 11 sub-components, which poses a problem of multiplicity.

\section{Conclusion}

The results of the CO-ILEPSS trial suggest that dual therapy with iloprost and eptifibatide for $48 \mathrm{~h}$ in patients with septic shock is safe and may even be beneficial. Biomarker measurements indicated reduced endothelial activation, disruption and cell damage, accompanied by less severe thrombocytopenia. Interestingly, the dual treatment resulted in significant reductions in SOFA score after $48 \mathrm{~h}$ compared to placebo treatment. Future adequately powered trials are warranted to reveal if this treatment may lead to improved patient-centred outcomes.

\section{Additional files}

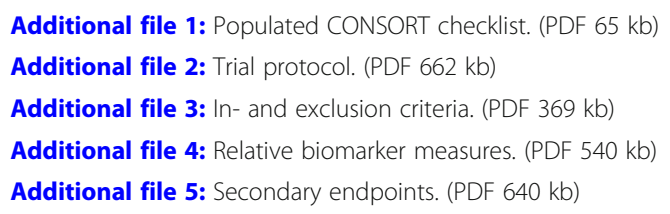

\section{Abbreviations}

CO-ILEPSS: Co-administration of iloprost and eptifibatide in septic shock; ELISA: Enzyme-linked immunosorbent assay; GP: Glycoprotein; ICU: Intensive care unit; ITT: Intention to treat; IQR: Interquartile range; LLD: Lower limit of detection; MHB: Morten Heiberg Bestle; NOH: Nordsjaellands Hospital; SOFA: Sequential Organ Failure Assessment; PACU: Post-anaesthesia care unit; PIJ: Pär Ivar Johansson; PP: Per protocol; REB: Rasmus Ehrenfried Berthelsen; RRT: Renal replacement therapy; SAE: Serious adverse event; SAPS II: Simplified Acute Physiology Score; SAR: Serious adverse reaction; SRO: Sisse Rye Ostrowski; sTM: Soluble thrombomodulin; SUSAR: Suspected unsuspected serious adverse reaction; sVEGFR1: Soluble vascular endothelial growth factor receptor 1; VEGF: Vascular endothelial growth factor

\section{Acknowledgements}

We would like to acknowledge all the nurses and doctors at the intensive care unit and the nurses at the post-operative care unit at NOH for the work associated with the trial treatment including randomisation, preparation of trial medication and blood sampling.

\section{Authors' contributions}

REB and SRO wrote the paper in collaboration. REB and MHB were the primary investigators and handled all patient screening, inclusion, trial treatment and data collection. SRO and PI wrote the protocol and performed the data analyses. All authors reviewed and approved the final version of the manuscript.

\section{Funding}

Funding was provided by Professor Per I. Johansson, Senior Physician, DMSC, MPA, The Capital Region Blood Bank, and Rigshospitalet. 


\section{Availability of data and materials}

The datasets used and analysed during the current study are available from the corresponding author on reasonable request.

\section{Ethics approval and consent to participate}

The CO-ILEPSS trial was approved by the ethics committee in The Capital Region of Denmark with protocol number: H-3-2014-087.

\section{Consent for publication}

Not applicable.

\section{Competing interests}

PIJ and SRO hold patent rights covering the use of prostacyclin and eptifibatide to patients with acute critical illness. All other authors declare that they have no competing interests.

\section{Author details}

'Department of Anesthesiology and Intensive Care, Zealand University Hospital, Roskilde, University of Copenhagen, Copenhagen, Denmark. ${ }^{2}$ Department of Clinical Immunology, Copenhagen University Hospital, Rigshospitalet, Blegdamsvej 9, 2100 Copenhagen $\varnothing$, Denmark. ${ }^{3}$ Department of Clinical Medicine, University of Copenhagen, Blegdamsvej 3B, 2200 Copenhagen N, Denmark. ${ }^{4}$ Section for Transfusion Medicine, Capital Region Blood Bank, Rigshospitalet, Blegdamsvej 9, 2100 Copenhagen $\varnothing$, Denmark. ${ }^{5}$ Center for Systems Biology, University of Iceland, Sturlugata 8, 101 Reykjavik, Iceland. ${ }^{6}$ Center for Translational Injury Research (CeTIR), Department of Surgery, University of Texas Health Science Center at Houston, 7000 Fannin, Suite 1800, Houston, TX 77030, USA.

Received: 23 May 2019 Accepted: 19 August 2019 Published online: 05 September 2019

\section{References}

1. Shankar-Hari M, Phillips GS, Levy ML, Seymour CW, Liu VX, Deutschman CS, et al. Developing a new definition and assessing new clinical criteria for septic shock. JAMA. 2016;315:775. https://doi.org/10.1001/jama.2016.0289.

2. Annane D, Aegerter P, Jars-Guincestre MC, Guidet B. Current epidemiology of septic shock: the CUB-Réa network. Am J Respir Crit Care Med. 2003;168: 165-72. https://doi.org/10.1164/rccm.2201087

3. Rhodes A, Bs MB, Co-chair R, Evans LE, Co-chair F, Alhazzani W, et al. Surviving sepsis campaign : international guidelines for management of sepsis and septic shock : 2016; 2017. https://doi.org/10.1097/CCM. 0000000000002255 .

4. Nguyen HB, Rivers EP, Abrahamian FM, Moran GJ, Abraham E, Trzeciak S, et al. Severe sepsis and septic shock: review of the literature and emergency department management guidelines. Ann Emerg Med. 2006;48:54.e1. https://doi.org/10.1016/J.ANNEMERGMED.2006.02.015.

5. Angus DC, van der Poll T. Severe sepsis and septic shock. N Engl J Med. 2013;369:840-51. https://doi.org/10.1056/NEJMra1208623.

6. Aird WC. The role of the endothelium in severe sepsis and multiple organ dysfunction syndrome. Blood. 2003;101:3765-77. https://doi.org/10.1182/ blood-2002-06-1887.

7. Karimova A, Pinsky DJ. The endothelial response to oxygen deprivation: biology and clinical implications. Intensive Care Med. 2001;27:19-31.

8. Boomer JS, To K, Chang KC, Takasu O, Osborne DF, Walton AH, et al. Immunosuppression in patients who die of sepsis and multiple organ failure. JAMA. 2011;306:2594-605. https://doi.org/10.1001/jama.2011.1829.

9. Esmon CT. Protein C pathway in sepsis. Ann Med. 2002;34:598-605.

10. Afshari A, Wetterslev J, Brok J, Møller AM. Antithrombin III for critically ill patients. In: Afshari A, editor. Cochrane database of systematic reviews. Chichester: Wiley; 2008. p. CD005370. https://doi.org/10.1002/14651858.CD005370.pub2.

11. Abraham E, Reinhart K, Opal S, Demeyer I, Doig C, Rodriguez AL, et al. Efficacy and safety of tifacogin (recombinant tissue factor pathway inhibitor) in severe sepsis. JAMA. 2003;290:238. https://doi.org/10.1001/jama.290.2.238.

12. Abraham E, Laterre P-F, Garg R, Levy H, Talwar D, Trzaskoma BL, et al. Drotrecogin alfa (activated) for adults with severe sepsis and a low risk of death. N Engl J Med. 2005;353:1332-41. https://doi.org/10.1056/NEJMoa050935.

13. Bernard GR, Vincent J-L, Laterre P-F, LaRosa SP, Dhainaut J-F, LopezRodriguez A, et al. Efficacy and safety of recombinant human activated protein C for severe sepsis. N Engl J Med. 2001;344:699-709. https://doi. org/10.1056/NEJM200103083441001.
14. Chen D, McVey JH, Dorling A. Enhanced effect of inhibition of thrombin on endothelium in murine endotoxaemia: specific inhibition of thrombocytopenia. Thromb Res. 2013;132:750-6. https:/doi.org/10.1016/j.thromres.2013.10.002.

15. Davies MG, Hagen PO. The vascular endothelium. A new horizon. Ann Surg. 1993;218:593-609.

16. Grande PO, Möller AD, Nordström CH, Ungerstedt U. Low-dose prostacyclin in treatment of severe brain trauma evaluated with microdialysis and jugular bulb oxygen measurements. Acta Anaesthesiol Scand. 2000;44:886-94.

17. Naredi S, Olivecrona M, Lindgren C, Ostlund AL, Grände PO, Koskinen LO. An outcome study of severe traumatic head injury using the "Lund therapy" with low-dose prostacyclin. Acta Anaesthesiol Scand. 2001;45:402-6.

18. Goerge T, Ho-Tin-Noe B, Carbo C, Benarafa C, Remold-O'Donnell E, Zhao B$\mathrm{Q}$, et al. Inflammation induces hemorrhage in thrombocytopenia. Blood. 2008;111:4958-64. https://doi.org/10.1182/blood-2007-11-123620.

19. Xiao Z, Théroux P. Frojmovic M. Modulation of platelet-neutrophi interaction with pharmacological inhibition of fibrinogen binding to platelet GPIIb/Illa receptor. Thromb Haemost. 1999;81:281-5.

20. Walther A, Czabanka M, Gebhard MM, Martin E. Glycoprotein IIB/IIIAinhibition and microcirculatory alterations during experimental endotoxemia - an intravital microscopic study in the rat. Microcirculation. 2004;11:79-88. https://doi.org/10.1080/10739680490266216.

21. Pu Q, Wiel E, Corseaux D, Bordet R, Azrin MA, Ezekowitz MD, et al. Beneficial effect of glycoprotein Ilb/Illa inhibitor (AZ-1) on endothelium in Escherichia coli endotoxin-induced shock. Crit Care Med. 2001;29:1181-8. https://doi. org/10.1097/00003246-200106000-00019.

22. Akcay AB, Yuce M, Akcay M, Sen N, Soydinc HE, Davutoglu V. Partial thrombus resolution with trofiban in a pregnant woman with mechanical prosthetic mitral valve thrombosis. Clin Appl Thromb. 2011;17:476-9. https://doi.org/10.1177/1076029610375422.

23. Yuce M, Davutoglu V, Sari I, Cakici M, Ercan S. Complete thrombus resolution with tirofiban in obstructive mechanical prosthetic mitral valve thrombosis. Platelets. 2010;21:386-8. https://doi.org/10.3109/09537101003768201.

24. Schulz KF. CONSORT 2010 statement: updated guidelines for reporting parallel group randomized trials. Ann Intern Med. 2010;152:726. https://doi. org/10.7326/0003-4819-152-11-201006010-00232.

25. Johansson PI, Mortensen CR, Nielsen T, Tollund C, Stensballe J, Hansen CP, et al. The effect of intraoperative and 6-h postoperative intravenous administration of low-dose prostacyclin on the endothelium, hemostasis, and hemodynamics in patients undergoing a pancreaticoduodenoctemy: a randomized-controlled pilot study. Eur J Gastroenterol Hepatol. 2017;29: 400-6. https://doi.org/10.1097/MEG.0000000000000800.

26. Holmvang L, Ostrowski SR, Dridi NP, Johansson P. A single center, open, randomized study investigating the clinical safety and the endothelial modulating effects of a prostacyclin analog in combination with eptifibatide in patients having undergone primary percutaneous coronary intervention (PCI) for ST-segment elevation myocardial infarction. Prostaglandins Other Lipid Mediat. 2012;99:87-95. https://doi.org/10.1016/j.prostaglandins.2012.08.002.

27. Zardi EM, Zardi DM, Cacciapaglia F, Dobrina A, Amoroso A, Picardi A, et al. Endothelial dysfunction and activation as an expression of disease: role of prostacyclin analogs. Int Immunopharmacol. 2005;5:437-59. https://doi.org/1 0.1016/J.INTIMP.2004.10.016.

28. Powell M, Mathru M, Brandon A, Patel R, Frölich M. Assessment of endothelial glycocalyx disruption in term parturients receiving a fluid bolus before spinal anesthesia: a prospective observational study. Int J Obstet Anesth. 2014;23:330-4. https://doi.org/10.1016/j.ijoa.2014.06.001.

29. Johansen $M$, Johansson P, Ostrowski S, Bestle M, Hein L, Jensen A, et al. Profound endothelial damage predicts impending organ failure and death in sepsis. Semin Thromb Hemost. 2015;41:016-25. https://doi.org/10.1055/s-0034-1398377.

30. Link A, Girndt M, Selejan S, Rbah R, Böhm M. Tirofiban preserves platelet loss during continuous renal replacement therapy in a randomised prospective open-blinded pilot study. Crit Care. 2008;12:R111. https://doi.org/10.1186/cc6998.

31. Neumann FJ, Zohlnhöfer D, Fakhoury L, Ott I, Gawaz M, Schömig A. Effect of glycoprotein $\mathrm{Ilb} / \mathrm{lll}$ a receptor blockade on platelet-leukocyte interaction and surface expression of the leukocyte integrin Mac-1 in acute myocardial infarction. J Am Coll Cardiol. 1999;34:1420-6. https://doi.org/10.1016/ S0735-1097(99)00350-2.

\section{Publisher's Note}

Springer Nature remains neutral with regard to jurisdictional claims in published maps and institutional affiliations. 